\title{
¿Decadal Variability in the North Pacific and North Atlantic under Global Warming: The Weakening Response and Its Mechanism
}

\author{
SHENG WU \\ LACOAS, Department of Atmospheric and Oceanic Sciences, Peking University, Beijing, China \\ ZHENG-YU LIU \\ Atmospheric Science Program, Department of Geography, The Ohio State University, Columbus, Ohio
}

(Manuscript received 6 January 2020, in final form 6 August 2020)

\begin{abstract}
We investigate the response of decadal variability in the North Pacific and North Atlantic under global warming and its mechanism in this study. To do so, we use four models (BCC-CSM1-1, CCSM4, IPSL-CM5A-LR, and MPIESM-LR) that participated in phase 5 of the Coupled Model Intercomparison Project (CMIP5), focusing on three global warming scenarios (RCP2.6, RCP4.5, and RCP8.5). Our analysis shows that the intensified global warming leads to a decrease in amplitude of both the Pacific decadal oscillation (PDO) and Atlantic multidecadal variability (AMV), resulting in reduced decadal variability of sea surface temperature (SST) in both the North Pacific and North Atlantic. In comparison, interannual variability is less impacted by global warming and has a tendency to increase, which leads to a shift of spectral power from decadal toward interannual variability. We then show the weakening decadal variability is caused partly by the weakened forcing of atmospheric heat flux variability, and partly by the increased SST damping rate. In addition, an enhanced upper-ocean stratification under global warming also contributes to the acceleration of Rossby waves, and a shift of decadal variability spectral power toward a shorter period.
\end{abstract}

KEYWORDS: Atmosphere-ocean interaction; Model output statistics; Interdecadal variability

\section{Introduction}

Over the last century, global-mean surface temperature (GMST) continued to rise due to increased concentrations of greenhouse gases in the atmosphere, with a trend of $0.61^{\circ} \mathrm{C}$ $(100 \mathrm{yr})^{-1}$ from 1861 to 2000 (Folland et al. 2001). However, after the turn of the century, the change of GMST was less than $0.05^{\circ} \mathrm{C}$ between 2000 and 2010 (Watanabe et al. 2014). Some studies indicated that this warming hiatus may be caused by the negative phase of the Pacific decadal oscillation (PDO) (Kosaka and Xie 2013; Farneti 2017). They found that the GMST stops growing during the negative phase of the PDO and accelerates in the positive state. This suggests that natural decadal variability (Liu 2012) may be crucial for climate prediction for the next few decades (Hawkins and Sutton 2009). Therefore, it is important to understand how and why decadal variability may change under future global warming.

Previous analyses studied the response of decadal variability to global warming. Some studies suggested that, under global warming, the time scale and amplitude of the decadal variability in the North Pacific sea surface temperature (SST) (Fang et al. 2014; Wang and Li 2017; Li et al. 2019) and Atlantic meridional overturning circulation (AMOC) tend to decrease (Cheng et al. 2016; MacMartin et al. 2016; Armstrong et al. 2017). Most previous research studied the response under

\footnotetext{
¿ Denotes content that is immediately available upon publication as open access.
}

Corresponding author: Zhengyu Liu, liu.7022@osu.edu; Sheng Wu,wishwishwish@pku.edu.cn global warming in the Pacific and Atlantic separately. Besides, the length of the simulation output is usually around 100 years, which is not sufficiently long for a thorough study of the change in decadal variability. In addition, the response of decadal variability is usually studied alone, without considering the accompanying interannual variability. Finally, the physical mechanism for the weakening decadal variability is not fully understood. Some studies attributed the weakening and shortening of decadal variability to the increased stratification under global warming and the acceleration of Rossby waves (Fang et al. 2014; Ortega et al. 2012; Cheng et al. 2016). No thorough study on the forcing mechanism in these two oceans, however, has been carried out.

Expanding on our previous work on the response of decadal variability to weak global warming scenario RCP2.6 (Wu et al. 2018), we find that the time scales of the PDO and Atlantic multidecadal variability (AMV) decreased significantly, but their amplitude decreased only slightly. Here, we perform a systematic study of the response of decadal variability of both PDO and AMV to different warming scenarios (RCP2.6, RCP4.5, and RCP8.5) in four CMIP5 coupled climate models that have long simulations. The response of decadal variability is also compared with that of interannual variability to help us understand the mechanism at work. Our analysis shows a significant weakening of decadal variability under global warming in both the North Pacific and North Atlantic, consistent with previous findings. However, we find that interannual variability is not changed substantially under global warming and has a tendency to increase. Therefore, there is a robust shift of spectral power from decadal toward interannual variability. Previous works speculated that the increased upper-ocean stratification in response to global warming may accelerate 
TABLE 1. CMIP5 models used in this study. Time period for RCP runs is from 2005 to 2300.

\begin{tabular}{|c|c|c|c|c|c|}
\hline Climate model & Institution & Country & $\begin{array}{l}\text { Horizontal } \\
\text { resolution }\end{array}$ & $\begin{array}{l}\text { Preindustrial } \\
\text { length }(\mathrm{yr})\end{array}$ & $\begin{array}{l}\text { RCP runs } \\
\text { length }(\mathrm{yr})\end{array}$ \\
\hline BCC_CSM1.1 & Beijing Climate Center & China & $360 \times 232$ & 500 & 295 \\
\hline CCSM4 & $\begin{array}{l}\text { National Center for Atmospheric } \\
\text { Research }\end{array}$ & $\begin{array}{l}\text { United } \\
\text { States }\end{array}$ & $320 \times 384$ & 500 & 295 \\
\hline IPSL-CM5A-LR & Institute Pierre Simon Laplace & France & $182 \times 149$ & 1000 & 295 \\
\hline MPI-ESM-LR & $\begin{array}{l}\text { Max Planck Institute for } \\
\text { Meteorology }\end{array}$ & Germany & $256 \times 220$ & 1000 & 295 \\
\hline
\end{tabular}

the Rossby waves, leading to a shorter decadal variability in the Pacific (Fang et al. 2014) and AMOC (Cheng et al. 2016). Here, our analysis further suggests that the weakening of decadal variability is caused by the reduced atmospheric surface heat variability forcing and the increased ocean surface damping rate, in addition to the increased upper-ocean stratification and acceleration of Rossby waves.

The outline of the paper is as follows. In section 2, we describe the data and method used in this study. Both observed PDO and AMV are presented in section 3 as a reference. The changes of decadal variability in the models are discussed in sections 4 and 5 using different methods. The mechanism of the response of decadal variability is presented in section 6 . Conclusions are given in section 7 .

\section{Data and method}

\section{a. Data}

The U.S. NOAA's Extended Reconstruction of SST dataset (ERSST), version 4, which contains monthly mean value in 1900-2016 with a horizontal spatial resolution of $2^{\circ} \times 2^{\circ}$, is used as the benchmark. Then, we interpolate observation data into the grid of $3.75^{\circ} \times 3.75^{\circ}$ by using the bilinear interpolation. Four coupled climate models will be used because they have the outputs of long global warming projection simulations, which are needed for examining the change of decadal variability. These models are part of the World Climate Research Programme's CMIP5 multimodel dataset (Table 1) (http://www.ipcc-data.org/sim/gcm_monthly/AR5/ReferenceArchive.html). For each model, the preindustrial (PI) run is used as its control run. We examine the changes of both PDO and AMV in the control and the future projection simulations under three warming scenarios of representative concentration pathway (RCP2.6, RCP4.5, and RCP8.5). The SST, heat flux, and density fields of the models are interpolated onto the same grids $\left(3.75^{\circ} \times 3.75^{\circ}\right)$ for convenience of comparison with observations.

\section{b. Statistical methods}

We first derive the annual mean from the monthly SST data. The SST anomaly (SSTA) is then obtained by subtracting the long-term mean from the annual mean. We derive the decadal variability by a standard low-pass filter with the 3-yr running mean applied twice (Zhang et al. 1997). Interannual variability is then derived as the high-pass variability from the annualmean data by subtracting the low-pass data.
To study decadal variability under global warming, we compare the variability of years $1-200$ of the PI control run in each model with that of years 2101-2300 of each of the three RCP scenarios: weak warming RCP2.6, intermediate warming $\mathrm{RCP} 4.5$, and strong warming RCP8.5. We derive both PDO and AMV indices from each CMIP5 model. Note that our 200yr time series is the same in length as that used for the decadal variability study of the AMOC in Cheng et al. (2016), and is twice the length of PDO analyses in, for example, Yim et al. (2015), Wang and Li (2017), and Lin et al. (2018). The response of decadal variability is studied in terms of the power spectrum of the index. The change of variability is studied in terms of the standard deviation of the filtered data. In the calculation of standard deviation, partly for comparison with the observation and partly to increase the sample size, we separate each 200-yr variability into two 100-yr segments (i.e., 1-100 and 101-200 for PI control, and 2101-2200 and 2201-2300 from three RCP runs). (The results are similar if the entire 200 years is used.) We calculate the 100 -yr standard deviation at each point, and then calculate the field averages over the North Pacific and North Atlantic. The amplitude of the total variability, decadal variability, and interannual variability are calculated as the standard deviations for the low-pass and high-pass anomalies, respectively.

\section{Observations}

Natural decadal climate variability has been observed all over the world, especially in the North Pacific and North Atlantic. As benchmarks, we use the ERSST data to show the PDO and AMV in the observation. In our previous work (Wu et al. 2018), we used the Hadley Centre Sea Ice and Sea Surface Temperature dataset (HadISST) as the benchmark, and we obtained similar results by using different observed reconstructions.

The PDO is defined as the first leading EOF mode of the North Pacific SSTA, weighted by the square root cosine of latitude. The spatial pattern of the PDO (Fig. 1a) is obtained by regressing SSTA on the first principal component (PC) time series, which resembles an El Niño-Southern Oscillation (ENSO)-like pattern (Mantua et al. 1997; Zhang et al. 1997). The positive phase of the PDO has negative SSTA in the central North Pacific, surrounded by positive anomalies in the Alaska gyre, off California, and toward the tropics (Schneider and Cornuelle 2005). The maximum regression amplitude is in the central North Pacific, while the tropical regression amplitude is around $80 \%$ of the maximum. The PDO index is considered as the first PC time series (Fig. 1c) of the EOF, and has 
(a) ERSST-PDO

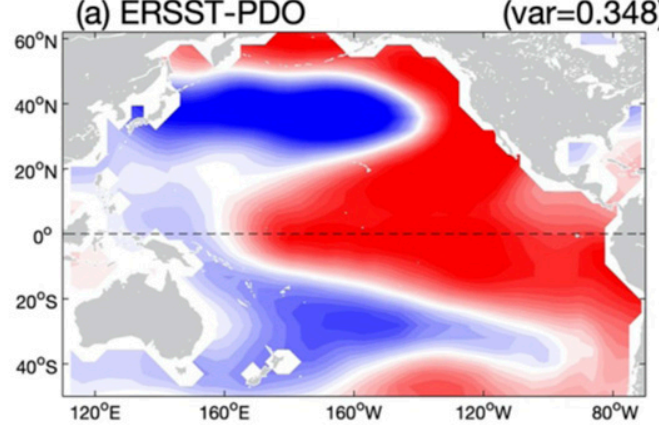

(c) ERSST index-PDO



(e) ERSST PowerSpec-PDO



(b) ERSST-AMV



(d) ERSST index-AMV

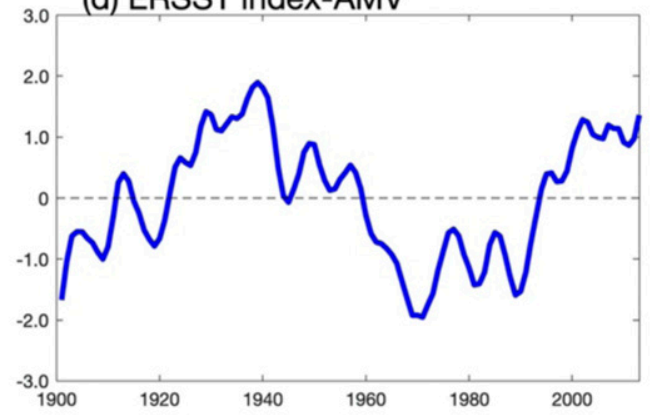

(f) ERSST PowerSpec-AMV

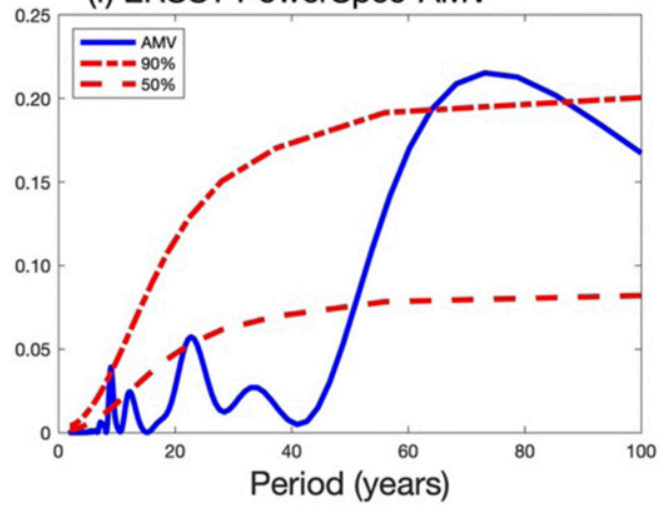

FIG. 1. Observed (top) spatial regression pattern, (middle) time series, and (bottom) power spectrum for (a),(c),(e) PDO and (b),(d),(f) AMV metrics (1900-2016). The PDO and AMV indices are calculated over the North Pacific $\left(20^{\circ}-70^{\circ} \mathrm{N}, 110^{\circ} \mathrm{E}-100^{\circ} \mathrm{W}\right)$ and North Atlantic $\left(0^{\circ}-70^{\circ} \mathrm{N}, 80^{\circ} \mathrm{W}-0^{\circ}\right)$, respectively. The dashed line and dash-dotted line in the bottom row represent $90 \%$ and $50 \%$ confidence intervals, respectively, calculated by the Monte Carlo method.

an apparent signal of decadal variability. A spectral analysis is then applied to the PDO index (Fig. 1e), with the red noise confidence level calculated by the Monte Carlo method.

The AMV is defined as the average of the North Atlantic SSTA. The AMV index (Fig. 1d) is derived by the mean of filed SSTA, weighted by the square root cosine of latitude. It is clear that the AMV phase changes on the multidecadal time scale, which is longer than that of the PDO. The AMV pattern (Fig. 1b) is obtained by the same method as the PDO pattern. The spatial pattern of the AMV is characterized by a horseshoe pattern (Deser and Blackmon 1993; Kushnir 1994). We also obtain the power spectrum for the AMV (Fig. 1f); and the results show that there are two peaks (around 20 and 70 years) in the North Atlantic. It is worth pointing out that the $20-\mathrm{yr}$ peak in the AMV is questionable as it only crosses the $50 \%$ confidence threshold.

\section{Response of decadal variability to global warming}

Here, we analyze the four CMIP5 coupled climate model simulations (Table 1) by using at least 200 years of each 


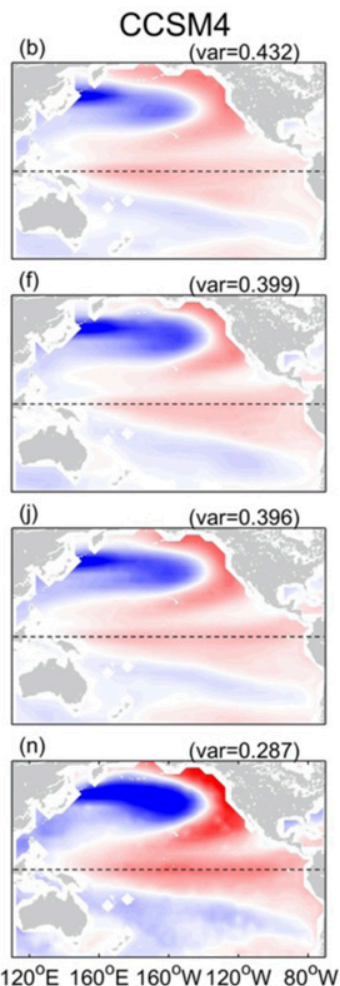
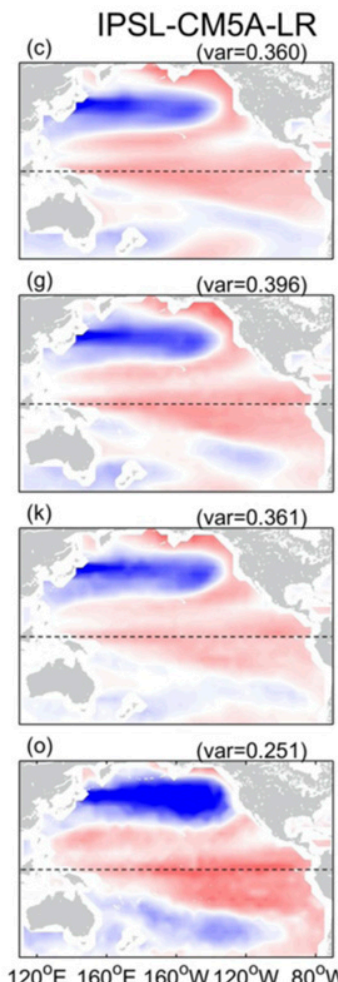

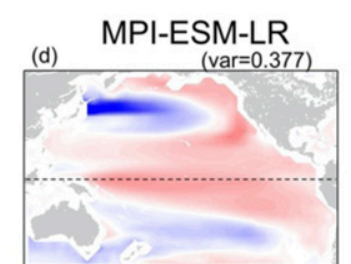

PI

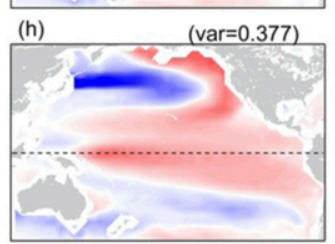

RCP26
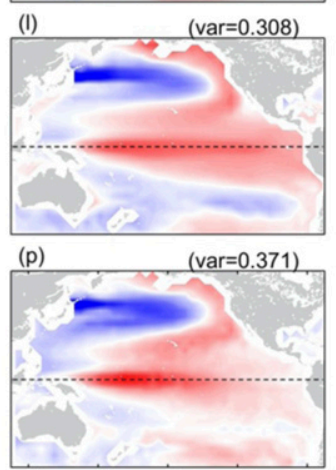

RCP85

FIG. 2. PDO spatial regression pattern from simulations of (top to bottom) the PI and three projected global warming scenarios in four CMIP5 models. The explained variance for the first EOF mode of North Pacific annual-mean SSTA is given at the upper right of each panel.

simulation output. Although this time period is still not sufficiently long for studying decadal variability with high statistical significance, it is adequate.

These four models reproduce the observed spatial patterns of PDO (Fig. 2) and AMV (Fig. 3) reasonably well in their PI runs. However, there are also some differences from the observations. The maximum of the Pacific regressing coefficient is in the central North Pacific in the observations, while it is in the Kuroshio-Oyashio Extension region in the CMIP5 climate models. In addition, the regression coefficients are smaller in the models than in the observation, especially in the tropics, reflecting the underestimated extratropical-tropical interaction in the models (Newman 2007). Under global warming, the patterns of the PDO and AMV do not show significant changes, consistent with previous findings (Overland and Wang 2007), while the explained variance for the first EOF mode of the North Pacific seems to show a decreasing trend under global warming except in the MPI model.

Under global warming, both PDO and AMV show a tendency of weakening amplitude and shorter period, consistent with previous findings (Fang et al. 2014; Cheng et al. 2016; Wu et al. 2018). We use the same method (see how we obtain PDO and AMV in section 3) to obtain the dominant time scale and spatial pattern of decadal variability for the PI run in the CMIP5 models. Then, we regress the three global warming scenarios SSTA on the PI's spatial pattern to obtain decadal variability time series. The PDO and AMV indices are calculated from a single 200-yr segment, and their power spectra are then calculated. The ensemble average of the power spectra is used for analyzing the changes of PDO and AMV under different global warming scenarios. The ensemble average of PDO and AMV power spectra for the PI, RCP2.6, RCP4.5, and RCP8.5 are shown in Figs. 4a and 4b, respectively. Visual comparison of the spectra between the PI and three RCP runs shows clearly a reduced decadal variability power, and a shift of spectral peaks from decadal toward higher frequency. For the PDO, the peaks of the PI are around 10, 20, and 40 years. Under global warming, the peaks tend to shift to shorter periods. We note that in this work the reduction in period of the PDO and AMV is in SST and surface climate variables. The shortening of AMV period is consistent with the shortening of the major period of AMOC that has been discussed in Cheng et al. (2016). Therefore, our study suggests that the response of AMOC under global warming may be related to SST and surface climate variables over the Atlantic.

Due to the short data length, the power spectral analysis on decadal and longer time scale variability is likely to be subject to substantial uncertainties. Therefore, we will assess the robustness of weakening decadal variability using the standard deviation of the SST variability in each basin. Now, the intensity of SSTA variability is represented by the SSTA standard deviation, which is averaged in the North Pacific and North Atlantic, respectively (Figs. 4c,d). The low-pass SSTA standard deviation is called decadal variability (left columns), 

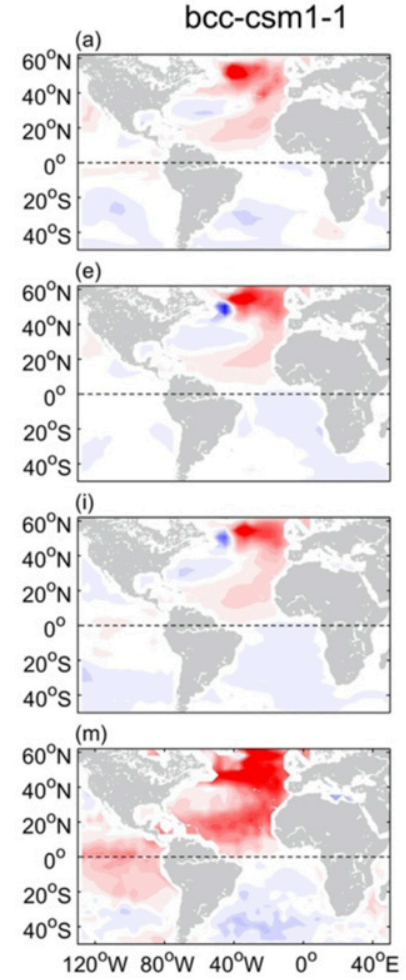




(g)
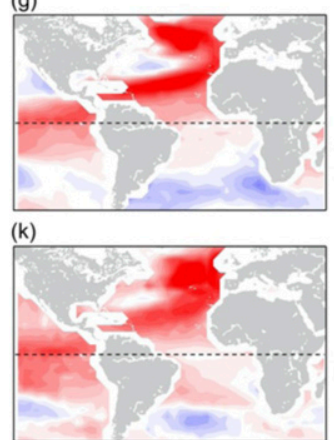

(o)

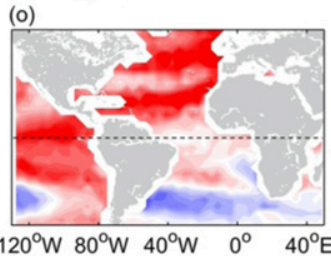

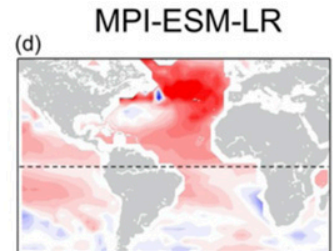

PI
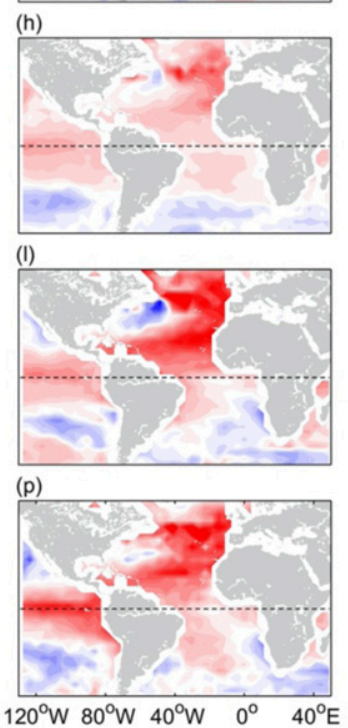

RCP26

RCP45

RCP85

FIG. 3. As in Fig. 4, but for AMV spatial regression pattern.

and the high-pass SSTA (the annual SSTA data minus the lowpass SSTA) standard deviation is called interannual variability (right columns). (The color for each scenario is the same as that for the power spectrum in Figs. 4a,b.) In comparison with observation (the purple line in Figs. 4c,d), model variability is much stronger for interannual variability but comparable for decadal variability in both oceans. In the North Pacific and North Atlantic, decadal variability is weakened under global warming, as seen in the reduction of standard deviation of the decadal variability from the PI to RCP8.5 (left columns in Figs. 4c,d). In the North Pacific, the median of decadal variability strength is reduced from 0.27 in the PI to 0.17 in RCP8.5, almost a $40 \%$ reduction, and in the North Atlantic, the median decadal variability is reduced from 0.24 to 0.15 , also about $40 \%$ reduction. In comparison with the decadal variability, however, interannual variability is reduced only slightly (right columns in Figs. 4c,d), in both oceans. The median interannual variability is reduced from 0.26 to 0.22 in the Pacific, and from 0.22 to 0.18 in the Atlantic, or about $15 \%$ reduction in both oceans, less than half of that for the decadal variability. Therefore, under global warming the spectral power of variability is shifted toward higher frequency, or from decadal variability toward interannual variability, in the models. This is consistent with the spectral analysis in Figs. $4 \mathrm{a}$ and $4 \mathrm{~b}$. It is interesting to note, however, that the weakening of variability under global warming is not monotonic, with relatively little change from RCP2.6 to RCP4.5 in both oceans for both decadal and interannual variability.
In sum, the analysis above on the ensemble of the four CMIP5 models suggests that the decadal variability weakens with the strength of global warming significantly. Furthermore, its weakening rate is faster than that of interannual variability. This result is robust in each individual model and insensitive to the filter length, as discussed below.

\section{Response of variability at different time scales}

In the analysis above, the low-pass decadal variability and the high-pass interannual variability are derived using the standard filter, in which the low-pass variability is derived by applying a 3-yr running mean twice and the high-pass interannual variability is derived as the residual. Given the limited length of the data, however, it is necessary to assess the robustness of our conclusion on low-pass and high-pass variability using different length of the filter.

First, we use a single 6-yr running mean $\left(\tau_{\mathrm{RM}}=6\right.$ years $)$ as the low-pass filter. This is similar to the standard filter, and therefore we expect that the result will remain the same. This is indeed the case. As in the standard filter in Fig. 4c, the ensemble mean standard deviation of the four models shows a significant decrease in the North Pacific (black in Fig. 5a), with little change between RCP2.5 and RCP 4.5. The interannual variability of the ensemble mean weakens less, and shows a slight increase (black in Fig. 5b). As a result, the ratio of decadal variability over interannual variability decreases monotonically (black in Fig. 5c), suggesting a robust reduction of decadal variability relative to interannual variability, and in 

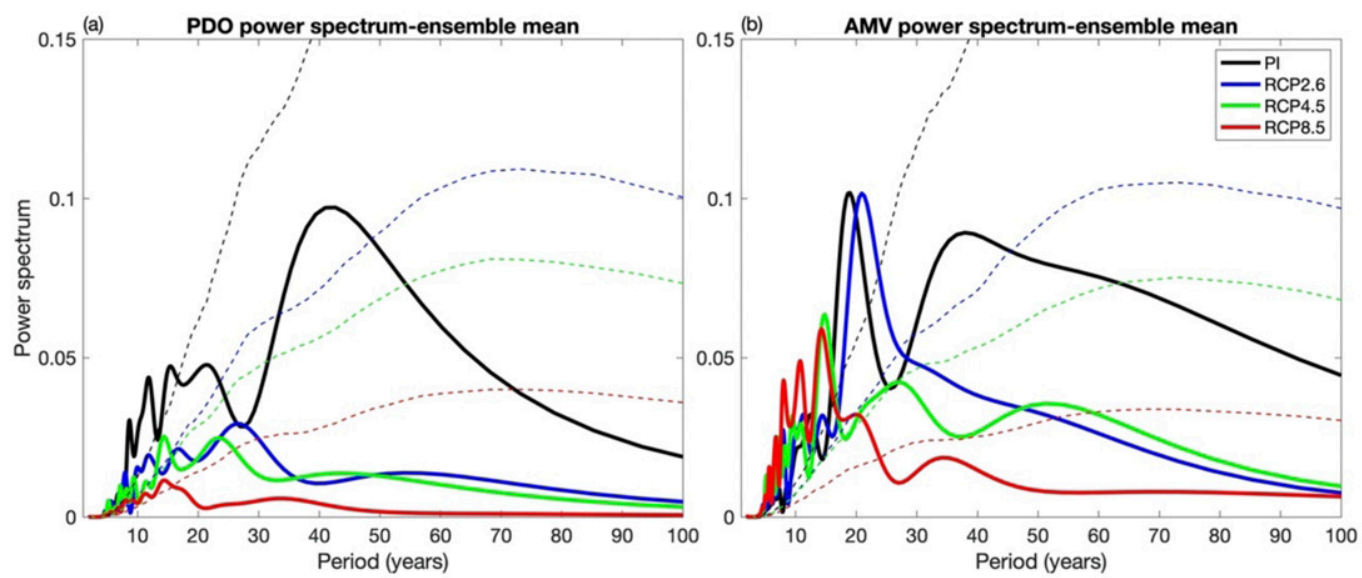

(c) NP SST STD



(d) NA SST STD

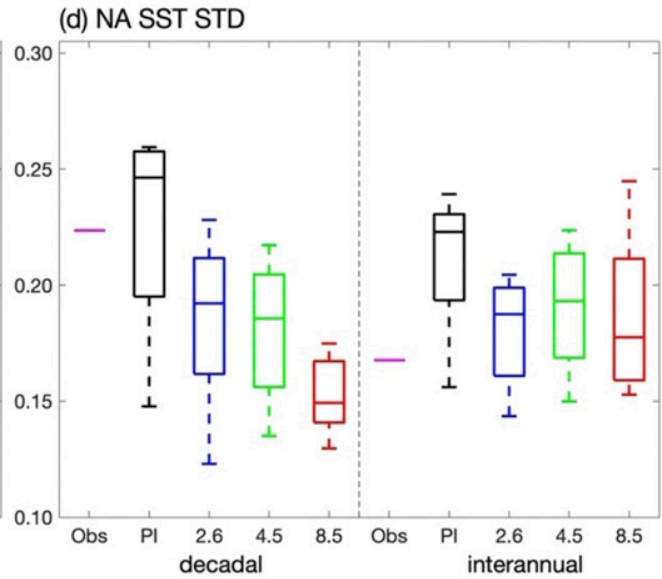

FIG. 4. (top) Ensemble-mean power spectra of (a) PDO and (b) AMV from the simulations of the PI and three projected global warming scenarios by the four CMIP5 models. The dashed lines represent the $90 \%$ confidence interval calculated by the Monte Carlo method (calculated for each member first and then obtained the ensemble mean confidence interval). (bottom) Distribution of the average of the (c) North Pacific and (d) North Atlantic SSTA standard deviation fields in observation, PI, and three projected global warming scenarios. "Decadal" and "interannual" mean decadal and interannual standard deviations, respectively. Boxplot boundaries are set at the 25 th and 75 th percentiles (p25 and p 75, respectively).

turn a spectral power shift from decadal to interannual variability. Furthermore, this response here can be seen in each individual model. The decadal variability weakens robustly across all four models from the PI to the strong warming scenario of RCP8.5 (color lines in Fig. 5a), although decadal variability increases slightly from RCP2.6 to RCP4.5 in CCSM4 and from the PI to RCP4.5 in IPSL. In comparison, the interannual variability decreases less from the PI to RCP8.5, and even increases monotonically in MPI (yellow line in Fig. 5b). This relative change of decadal variability shows clearly a faster decrease of decadal variability than interannual variability in the three models, almost monotonically under global warming. The IPSL model seems to be an exception, which increases slightly from the PI to RCP4.5 and then decreases sharply to RCP8.5 (green line in Fig. 5c). An overall similar response is also found in the North Atlantic (Figs. 6a-c).

These responses of the magnitude of low-pass ("decadal") and high-pass ("interannual") variability, and their differences, can be seen more systematically using different filtering time $\tau_{\mathrm{RM}}$. Now, we calculate the weakening trend of low-pass variability in the Pacific as the slope of standard deviation from the PI, to RCP2.6, RCP4.5, and RCP8.5, and plot the weakening rate as a function of $\tau_{\mathrm{RM}}$ in Fig. $5 \mathrm{~d}$, for each model and ensemble mean using the same color as in Fig. 5a. For example, the slope of the ensemble-mean low-pass variability (solid black line in Fig. 5a) corresponds to the intersection point between the black solid line and dashed line in Fig. $5 d\left(\tau_{\mathrm{RM}}=\right.$ 6 years). A negative slope represents a standard deviation decreasing under global warming, or the weakening trend of low-pass variability as discussed in Fig. 5a. First, the slope of low-pass variability remains negative for $\tau_{\mathrm{RM}}$ up to 30 years for the ensemble mean and individual models (Fig. 5d). The most negative slope occurs for $\tau_{\mathrm{RM}}$ of at 6 years, and is reduced slightly at the small $\tau_{\mathrm{RM}}$ limit, $\tau_{\mathrm{RM}}=1$ year, in which the low-pass variability becomes the total variability (stars at $\tau_{\mathrm{RM}}=1$ year). With increasing $\tau_{\mathrm{RM}}$, the negative slope decreases, which is caused, at least partly, by the reduction of variability spectral power 
(a) NP SST decadal STD

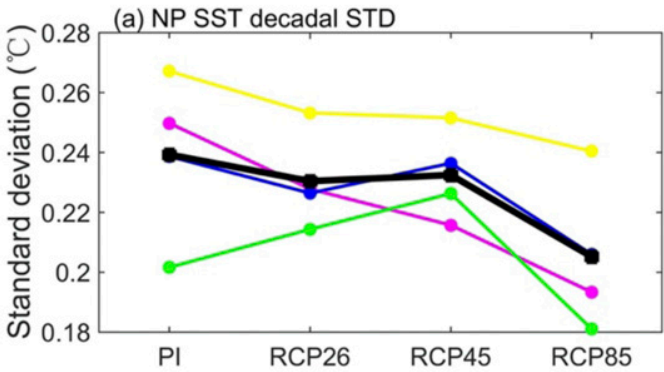

(b) NP SST interannual STD

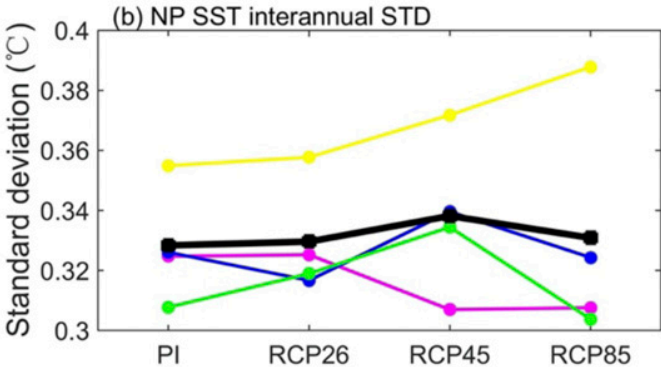

(c) NP SST decadal/interannual STD



(d) NP Low Frequency



(e) NP High Frequency
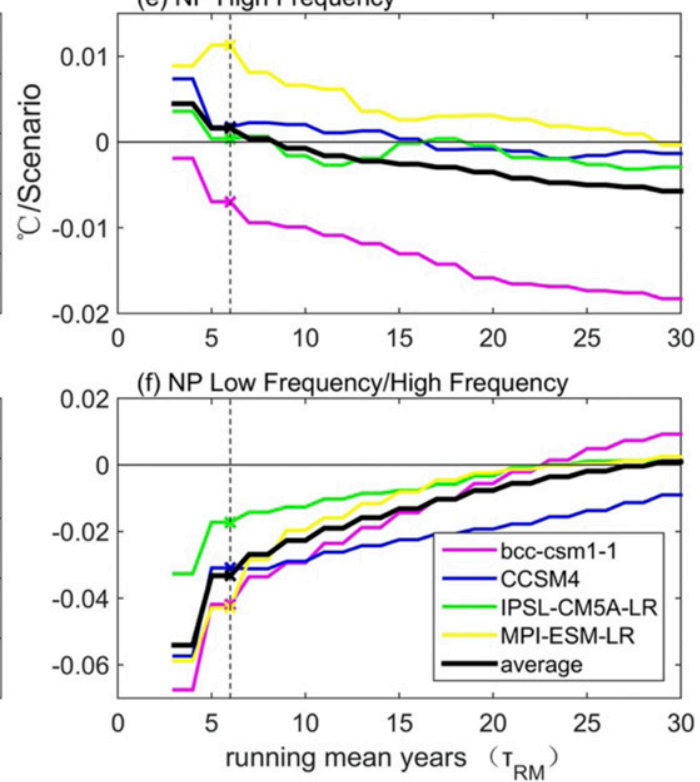

FIG. 5. The change of SSTA standard deviation in the North Pacific. We used a 6-yr running mean to obtain the low-pass data and calculated field average of the (a) decadal, (b) interannual, and (c) ratio of decadal to interannual standard deviations. (d)-(f) We used different running mean years to obtain low- and highfrequency standard deviation changes under global warming. The pentacle marks in (d) represent the slope of the total variability.

toward very low frequency. Therefore, the weakening trend of the low-pass variability is robust for $\tau_{\mathrm{RM}}$ up to 30 years, and the strongest weakening trend occurs for $\tau_{\mathrm{RM}}$ of $\sim 6$ years. In contrast, the slope of high-pass variability is positive for $\tau_{\mathrm{RM}}$ smaller than $\sim 6$ years (except for BCC model) and decreases to a negative slope with further increase of $\tau_{\mathrm{RM}}$ (Fig. 5e). Therefore, high frequency is intensified with global warming, opposite to low-frequency decadal variability. As $\tau_{\mathrm{RM}}$ further increases, the slope becomes negative, because the high-pass variability now starts to include longer term decadal variability. At the large $\tau_{\mathrm{RM}}$ limit, the high-pass variability approaches the total variability, and therefore the slope (Fig. 5e) becomes similar to that of the small $\tau_{\mathrm{RM}}$ limit of low-pass variability, or total variability (Fig. 5d), as a strong weakening trend. The slope of the ratio between low-pass and high-pass variability is always negative (except in BCC for longer than 20 years) (Fig. 5f), suggesting a stronger decreasing trend of low-pass relative to high-pass variability, insensitive to the filter length $\tau_{\mathrm{RM}}$, and therefore a shift of low-frequency variability spectral power toward higher-frequency variability.

The result in the North Atlantic is overall similar (Figs. 6d-f). One difference from the Pacific is that the $\tau_{\mathrm{RM}}$, at which the slope of low-pass variability maintains its most negative value and at which the slope of high-pass variability becomes positive, seems to be extended to $\sim 10$ years, at least for the ensemble mean. The difference is that the slope of the high-pass standard deviation is always positive in CCSM4 and MPI models, suggesting that the high-pass variability, and even the total variability, which is dominated by interannual variability, increases with global warming. Partly, perhaps, this occurs because the spectral power of the AMV is concentrated more on the multidecadal variability beyond 30 years. This positive slope of highpass variability in CCSM4 and MPI models is also present in the Pacific, but decreases with $\tau_{\mathrm{RM}}$ and becomes negative after $\tau_{\mathrm{RM}}$ becomes longer than 15 years for CCSM4 (Fig. 5e).

Overall, our analysis suggests that the weakening of decadal variability under global warming is robust in the North Pacific 
(a) NA SST decadal STD

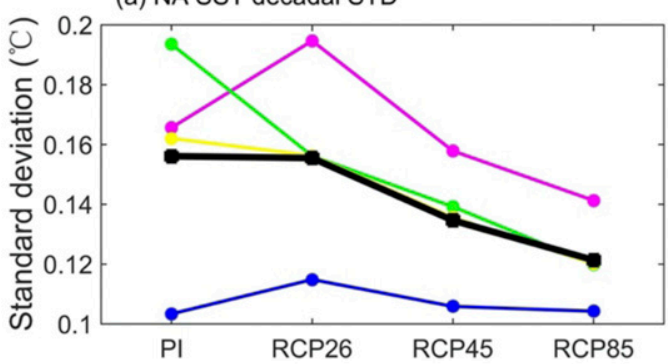

(b) NA SST interannual STD



(c) NA SST decadal/interannual STD

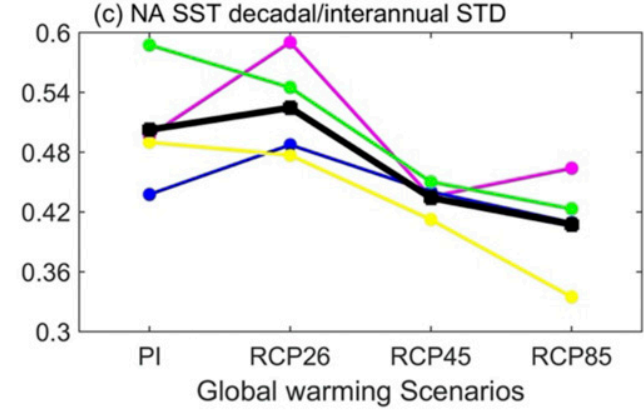

(d) NA Low Frequency



(e) NA High Frequency
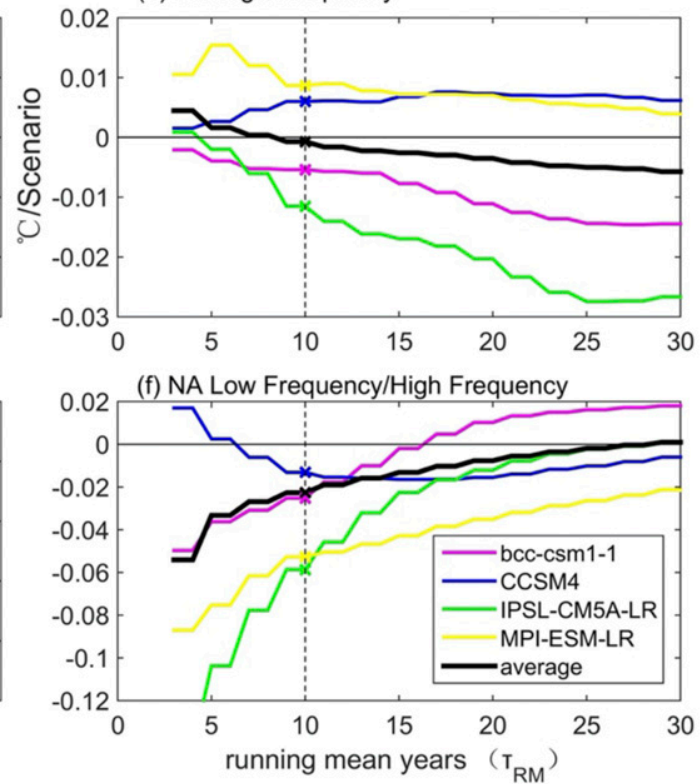

FIG. 6. As in Fig. 5, but for the North Atlantic.

and North Atlantic, and the interannual variability has a tendency to increase accordingly, such that the spectral power of variability shifts toward higher frequency with increasing global warming.

\section{Mechanism}

The robust weakening of decadal variability under global warming, and the spectral power shift toward interannual variability, in both the North Pacific and North Atlantic, could be associated with some common mechanism. We will analyze the mechanism of weakening decadal variability more systematically next.

First, we study the response of SST in the simplest framework: the local heat budget framework in a stochastic climate model (Hasselmann 1976):

$$
C_{P} \rho h \frac{d T}{d t}=H,
$$

where $H$ is the total surface heat flux, which can be decomposed into two parts:

$$
H=q-\mu_{A} T,
$$

where $q$ is the internal atmospheric variability that is independent of SSTA and $-\mu_{A} T$ is the negative oceanic feedback (Frankignoul et al. 1998). Using Eq. (1b), Eq. (1a) can be written as

$$
\frac{d T}{d t}=F-\lambda_{A} T
$$

with

$$
F=\frac{q}{C_{P} \rho h} \quad \text { and } \quad \lambda_{A}=\frac{\mu_{A}}{C_{P} \rho h},
$$

as the atmospheric forcing and local damping rate, respectively. The spectral power of the SST variability can therefore be derived from Eq. (2) using Fourier transformation as follows,

$$
|T(\omega)|=\frac{|F(\omega)|}{\sqrt{\omega^{2}+\lambda_{A}^{2}}} .
$$

The change of SST variability should be proportional to the heat flux forcing. A simple hypothesis is that the weakening decadal variability and the power spectra shift from decadal to 

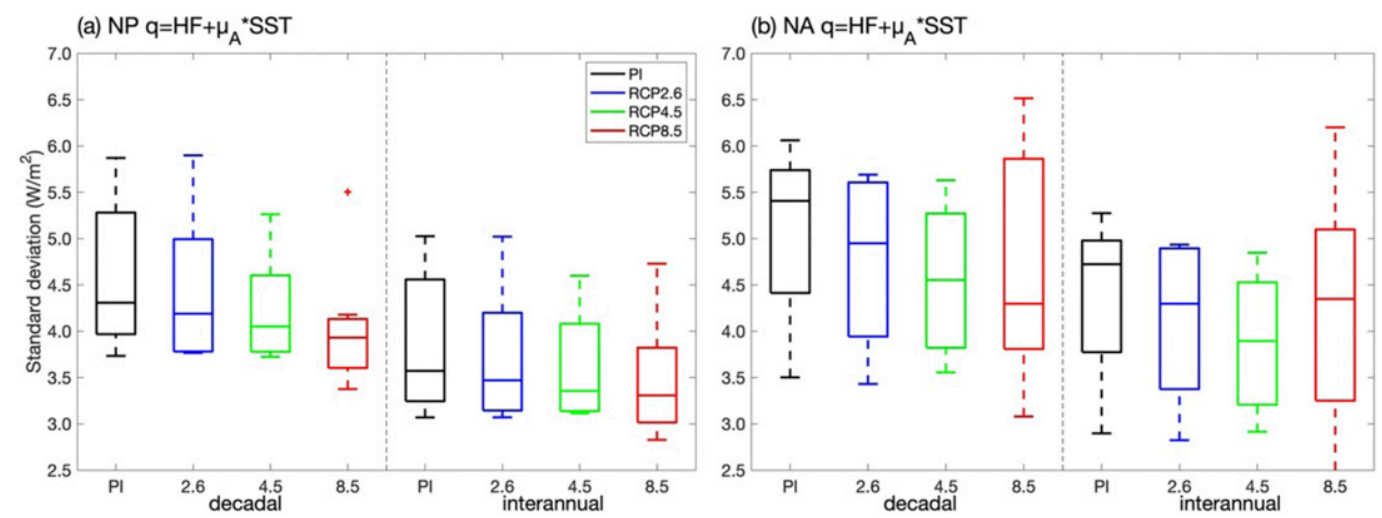

FIG. 7. Distributions of the average of the (a) North Pacific and (b) North Atlantic atmospheric forcing standard deviation fields in observation, PI, and three projected global warming scenarios. Boxplot boundaries are set at the 25th and 75th percentiles (p25 and p75, respectively).

interannual are caused simply by the weakening atmospheric heat flux forcing.

This simple hypothesis is only partly true. Using monthly data of total surface heat flux $H_{m}$ and SST $T_{m}$, where $m=1,2, \ldots$, the feedback parameter can be estimated using the lagged covariance as in Frankignoul et al. (1998):

$$
\mu_{A}=\left\langle H_{m}, T_{m+1}\right\rangle /\left\langle T_{m}, T_{m+1}\right\rangle .
$$

The heat flux induced by atmospheric internal variability can be derived as $q=H+\mu_{A} T$. For different global warming scenarios, the response of the heat flux variability can be separated into decadal (left) and interannual (right), as for the SST variability in Figs. 4c and 4d, respectively. At the first sight, in both oceans the heat flux variability weakens with global warming similar to the SST variability, with a stronger weakening for decadal variability and a weaker one for interannual variability. Therefore, the weakening of decadal variability and the shift of spectral power from decadal to interannual variability can be from the atmospheric internal variability and the associated heat flux forcing over the ocean. The cause of the change of atmospheric internal variability may be related to the increase of static stability (Frierson 2006).

A more careful comparison of the change of heat flux forcing and SST variability, however, reveals that the weakening of heat flux variability contributes less than half to the weakening of the SST variability. From the PI to RCP8.5, the median decadal heat flux standard deviation decreases from 4.3 to $3.8 \mathrm{~W} \mathrm{~m}^{-2}$ in the Pacific and from 5.4 to $4.3 \mathrm{~W} \mathrm{~m}^{-2}$ in the Atlantic (Fig. 7), giving reductions of $\sim 10 \%$ and $20 \%$, respectively. This is less than half of the $40 \%$ reduction in SST (see Figs. 4c,d). Similarly, interannual variability is reduced by about $5 \%$ and $8 \%$ in the Pacific and Atlantic, respectively, which is less than half of the $15 \%$ weakening in SST (in Figs. 4c,d). Therefore, the weakening of the SST variability with global warming has to come from other mechanisms.

One potential mechanism is the damping rate of SST. In Eq. (2), if nonlocal processes, subsurface processes, and entrainment processes are considered, they may also induce an additional damping $\lambda_{O}$ such that the SST equation becomes

$$
\frac{d T}{d t}=F-\lambda T
$$

with $\lambda=\lambda_{A}+\lambda_{O}$ as the total damping rate. The SST standard deviation now becomes

$$
|T(\omega)|=\frac{|F(\omega)|}{\sqrt{\omega^{2}+\lambda^{2}}} .
$$

This total damping rate can be estimated from SST autocorrelation coefficient $r$ as $\lambda=(1+r) / \Delta t$, with $\Delta t=1$ month. More specifically, following Frankignoul et al. (1998), we use a least squares fit over weighted lags of 1-3 months to obtain the ocean damping rate at each grid point. An increase of the total damping rate should cause a decrease in the SST variability. The damping rate estimated at each grid point and then averaged over the North Pacific and North Atlantic for each model and the model ensemble are shown in Figs. $8 \mathrm{a}$ and $8 \mathrm{~b}$, respectively. Overall, the damping rate is on the order of $\lambda \sim$ 0.2 month $^{-1}$. The damping rate indeed shows an increasing trend with global warming in both oceans. Although there is a larger spread, we can see a significant increase in RCP8.5. We also calculate the significance test at each point using the Monte Carlo method. Results suggest the increasing trend of damping rate is still valid (not shown). As a result, for lowfrequency variability of decadal time scale that satisfies the quasi-equilibrium condition:

$$
\omega \ll \lambda \sim 0.2 \text { month }^{-1} \text {, or period } \gg 5 \text { months, }
$$

the amplitude in Eq. (7) will decrease roughly as the quasiequilibrium temperature response $F / \lambda$, faster than the heat flux in Fig. 7. This faster weakening response of equilibrium temperature is shown clearly in Figs. $8 \mathrm{c}$ and $8 \mathrm{~d}$ [where $F$ is derived from a constant mixed layer $h=50 \mathrm{~m}$ and the estimated atmospheric heat flux $q$ as in Eq. (3)]. Now, the median standard deviation of decadal variability decreases from $0.4^{\circ} \mathrm{C}$ in the PI to $0.27^{\circ} \mathrm{C}$ in RCP8.5 in the Pacific, and 



FIG. 8. (top) The field average of ocean damping rate ( $\lambda$ ) in the (a) North Pacific and (b) North Atlantic. (bottom) Distributions of the average of the (c) North Pacific and (d) North Atlantic and the ratio of white noise $(F)$ and ocean damping rate in observation, PI, and three projected global warming scenarios. The error bars in (a) and (b) represent field standard deviations. Boxplot boundaries are set at the 25th and 75th percentiles (p25 and p75, respectively).

from $0.47^{\circ}$ to $0.3^{\circ} \mathrm{C}$ in the Atlantic (Figs. $8 \mathrm{c}, \mathrm{d}$, left columns), giving a weakening rate of about $35 \%$ in both oceans, faster than the heat flux weakening, and almost comparable with the SST weakening rate of $\sim 40 \%$. Also, interestingly, the damping rate does not increase from RCP2.6 to RCP4.5 (Figs. 8a,b), which also favors less weakening between these two scenarios as seen in the SST variability (Figs. 4c,d, left columns). One may also calculate the same equivalent temperature for interannual variability (Figs. 8c,d, right columns). This gives a weakening rate of almost $30 \%$, faster than that of SST (Figs. 4c,d, right columns). This is because interannual variability does not satisfy the quasi-equilibrium response condition of Eq. (8), and instead it should use the full solution in Eq. (7), in which an increasing damping rate reduces the temperature amplitude less than the equilibrium response. In other words, the equilibrium temperature overestimates the weakening rate for the interannual variability.

A further study is needed to understand why the total damping rate is increased with global warming. We speculate the increased damping rate with global warming may be caused by the faster surface warming, a shallow mixed layer and a stronger entrainment effect. As discussed in previous works, as global warming intensifies, the surface ocean warms up more than the subsurface, leading to a stronger stratification (Saenko 2006) (Fig. 9, first and third columns). The faster surface warming, we suggest, also reduces the mixed layer depth (Jang et al. 2011), as indicated in the evolution of vertical density profiles in the models (Fig. 9, second and fourth columns). The shallower mixed layer, we speculate, may increase the damping rate in several ways. First, it can increase the damping rate associated with the atmospheric feedback $\lambda_{A}$, for given feedback $\mu_{A}$, as shown in Eq. (3). If the mixed layer $h$ decreases, the atmospheric feedback $\lambda_{A}$ would be larger. Second, the shallower mixed layer may also render the damping associated with the entrainment effect more effective on SST.

Finally, the increased stratification with global warming may contribute to both the weakening of decadal variability and the shift of spectral power toward high-frequency variability, along 

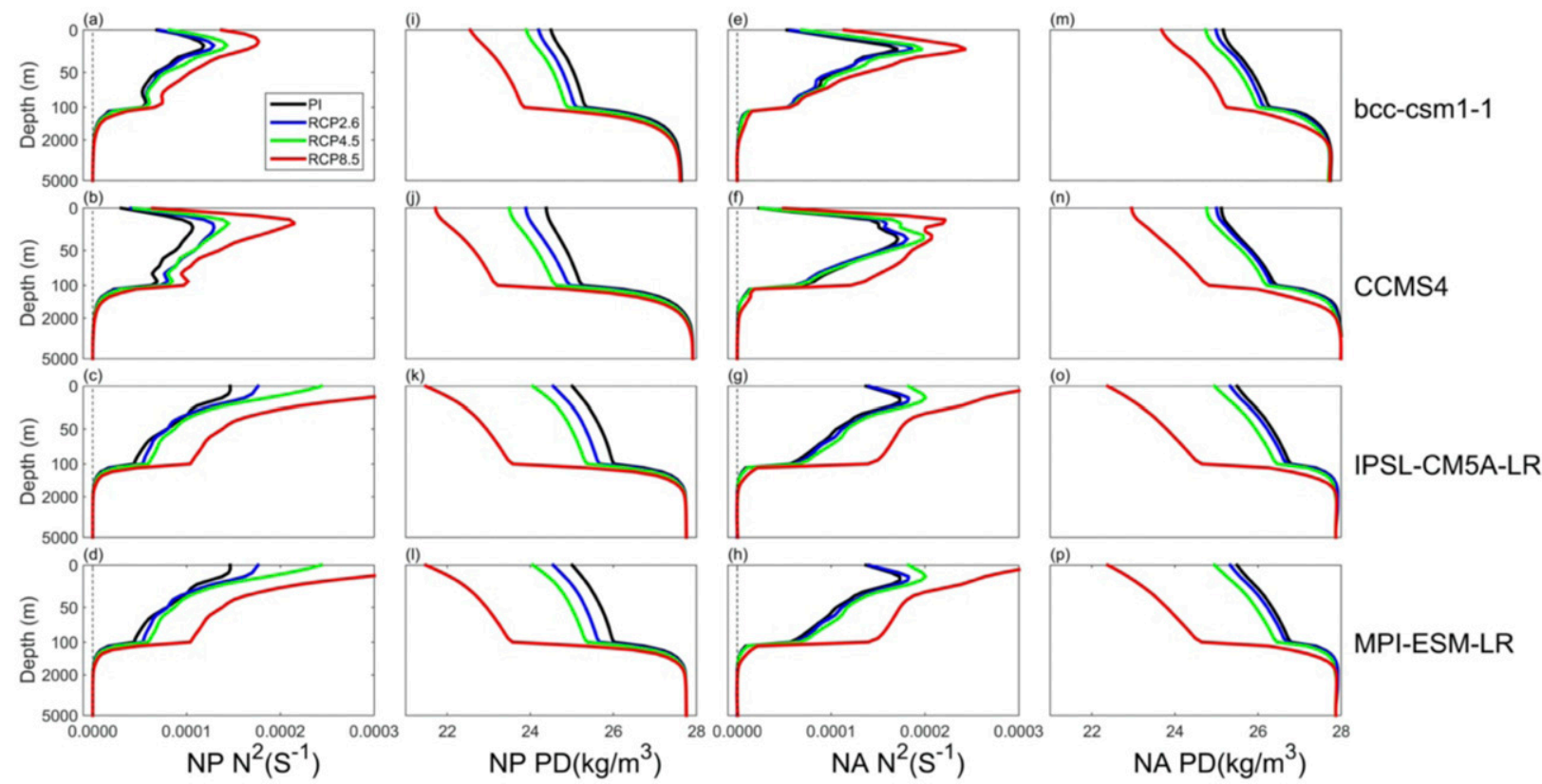

FIG. 9. Vertical profiles of area-mean (a)-(h) buoyancy frequency $\left(N^{2}\right)$ and (i)-(p) potential density of the (left two columns) North Pacific and (right two columns) North Atlantic in PI and three projected global warming scenarios of the four CMIP5 models: (top to bottom) BCC-CSM1-1, CCSM4, IPSL-CM5A-LR, and MPI-ESM-LR.

with an increased speed of Rossby wave propagation. If a wave propagation effect is added onto the SST Eq. (7), we have

$$
\frac{\partial T^{\prime}}{\partial t}-c \frac{\partial T^{\prime}}{\partial x}=F^{\prime}-\lambda T^{\prime}
$$

For a typical wavenumber $k$, we have $F^{\prime}=F(t) \exp (i k x)$, $T^{\prime}=T(t) \exp (i k x)$ with the amplitude of $T(t)$ satisfying

$$
\frac{d T}{d t}-i \omega_{0} T=F-\lambda T
$$

where $\omega_{0}=k c$. This gives the spectral power of the SST response as follows:

$$
|T(\omega)|=\frac{F(\omega)}{\sqrt{\left(\omega-\omega_{0}\right)^{2}+\lambda^{2}}} .
$$

Given the increased stratification with global warming, Rossby wave speed will be increased as $c \sim N^{2}$. This will increase $\omega_{0}$ and therefore the shift of power from low toward higher frequency, as the SST variability. Indeed, the Rossby wave speed is increased with intensified global warming, as calculated using continuously stratified quasigeostrophic eigenvalue equation (Fig. 10). Given a fixed $F$, a speed up of Rossby wave will lower the variance at lower frequency (decadal) and increase the variance at higher frequency (interannual). This relation between wave speed increase and amplitude reduction has been suggested in previous studies (Fang et al. 2014; Ortega et al. 2012; Cheng et al. 2016).

Here, we point out that if the increased stratification and the speed up of Rossby wave is one mechanism, it may not be induced by wind forcing; instead, it may be caused by surface heat flux forcing. For long Rossby waves, the continuously stratified quasigeostrophic equation forced by surface wind stress and heat flux forcing rate $Q$ is

$$
\partial_{t}\left[\partial_{z}\left(\frac{f_{0}^{2}}{N^{2}} \partial_{z} \varphi\right)\right] \varphi+\beta \partial_{x} \varphi=\frac{\operatorname{curl}\left(F_{\text {wind }}\right)}{\rho}+\partial_{z}\left(\frac{f_{0} \rho Q}{N^{2}}\right)
$$

where an increased stratification $N^{2}$ is seen to increase the wave speed: $c \sim \beta / \partial_{z}\left[\left(f_{0}^{2} / N^{2}\right) \partial_{z} \varphi\right]$.

In the low-frequency limit (applicable to decadal variability), the quasi-equilibrium response is further reduced to the generalized Sverdrup relation:

$$
\beta \partial_{x} \varphi=\frac{\operatorname{curl}\left(F_{\text {wind }}\right)}{\rho}+\partial_{z}\left(\frac{f_{0} \rho Q}{N^{2}}\right) .
$$

An increased $N^{2}$ is seen here to reduce the effect of surface buoyance forcing, which may also drive thermocline variability through ventilation effect and distorted potential vorticity (Liu and Pedlosky 1994). Physically, a stronger stratification will suppress the distortion on potential vorticity by surface heat flux forcing and in turn reduce thermocline variability. This is similar to MacMartin et al. (2016) results. Note, however, this suppression effect of stratification is not effective to wind forcing, because the effect of stratification against the wind forcing and Ekman pumping is canceled by that of the speed up of Rossby waves.

\section{Conclusions}

A systematic study on the response of decadal variability to global warming is performed for the North Pacific and North Atlantic using four CMIP5 climate models under three global 
(a) NP Rossby Wave

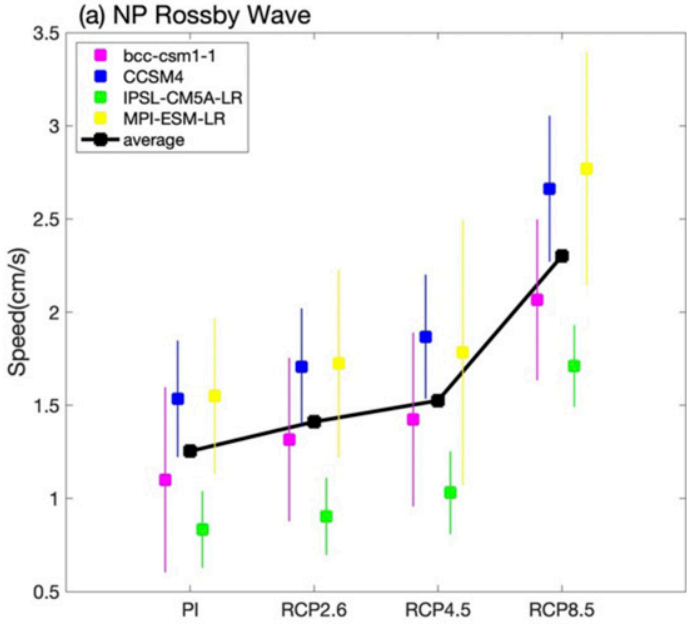

(b) NA Rossby Wave

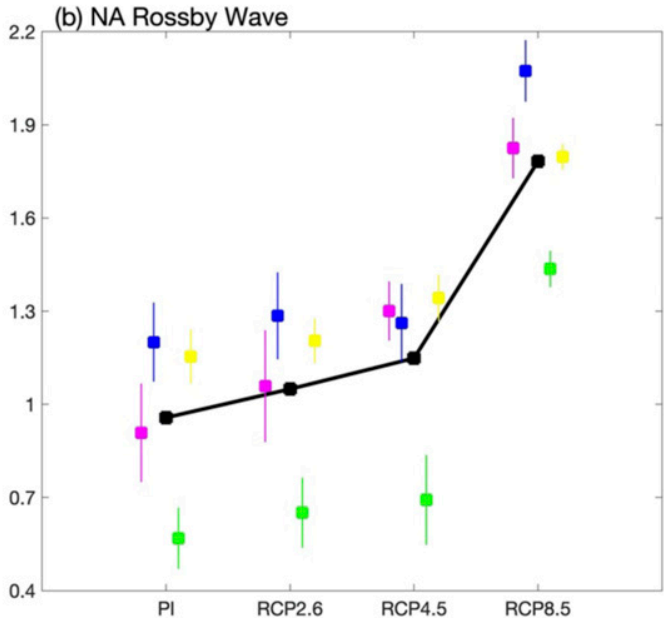

FIG. 10. Rossby wave speed of the (a) North Pacific (NP) and (b) North Atlantic (NA) in PI and three projected global warming scenarios of the four CMIP5 models. Filled squares show the change of Rossby wave speed under global warming. The error bars represent the field standard deviations.

warming scenarios. Consistent with previous findings (Fang et al. 2014; Cheng et al. 2016; MacMartin et al. 2016; Armstrong et al. 2017; Wang and Li 2017; Li et al. 2019), we find that a robust response of PDO and AMV to global warming is a weakening of decadal variability, especially under strong global warming. Furthermore, we find that interannual variability shows relatively less change and could even increase relative to the decadal variability under global warming. As a result, there is a robust spectral power shift from decadal variability toward interannual variability. This shift toward higher frequency is also consistent with the notion of a shortening time scale of the PDO and AMV. But the patterns of decadal variability are not changed. We further investigate the cause for the weakening of decadal variability and suggest several new mechanisms. For both the North Pacific and North Atlantic, the dominant mechanism for the reduction of decadal variability is the reduced stochastic atmospheric heat flux variability with global warming and the increased SST damping rate, each contributing to about half of the weakening of the decadal variability. Finally, the weakening can also be contributed by the intensified upper-ocean stratification with global warming in two ways. First, a stronger stratification may suppress the effect of surface heat flux forcing on thermocline variability. Second, the intensified stratification can also accelerate Rossby waves, which may also cause the shortening of time scale and a spectral power shift from decadal to higher frequency, as proposed in previous studies (e.g., Fang et al. 2014; Cheng et al. 2016; Li et al. 2019). The variability changes in the warming hiatus and the accelerating warming periods, which are related to the variability changes in different phases of decadal oscillation, are interesting, and need to be studied in the future.

Acknowledgments. This work is supported by Chinese MOST (2017YFA0603801), NSFC (41630527), and U.S. NSF
(AGS-1656907). The China Scholarship Council funded Wu's visit at the Georgia Institute of Technology.

\section{REFERENCES}

Armstrong, E., P. Valdes, J. House, and J. Singarayer, 2017: Investigating the impact of $\mathrm{CO}_{2}$ on low-frequency variability of the AMOC in HadCM3. J. Climate, 30, 7863-7883, https:// doi.org/10.1175/JCLI-D-16-0767.1.

Cheng, J., Z. Liu, S. Zhang, W. Liu, L. Dong, P. Liu, and H. Li, 2016: Reduced interdecadal variability of Atlantic meridional overturning circulation under global warming. Proc. Natl. Acad. Sci. USA, 113, 3175-3178, https://doi.org/10.1073/pnas.1519827113.

Deser, C., and M. L. Blackmon, 1993: Surface climate variations over the North Atlantic ocean during winter: 19001989. J. Climate, 6, 1743-1753, https://doi.org/10.1175/ 1520-0442(1993)006<1743:SCVOTN >2.0.CO;2.

Fang, C., L. Wu, and X. Zhang, 2014: The impact of global warming on the Pacific decadal oscillation and the possible mechanism. Adv. Atmos. Sci., 31, 118-130, https://doi.org/10.1007/s00376013-2260-7.

Farneti, R., 2017: Modelling interdecadal climate variability and the role of the ocean. Wiley Interdiscip. Rev.: Climate Change, 8, e441, https://doi.org/10.1002/wcc.441.

Folland, C. K., and Coauthors, 2001: Global temperature change and its uncertainties since 1861. Geophys. Res. Lett., 28, 26212624, https://doi.org/10.1029/2001GL012877.

Frankignoul, C., A. Czaja, and B. L'Heveder, 1998: Air-sea feedback in the North Atlantic and surface boundary conditions for ocean models. J. Climate, 11, 2310-2324, https://doi.org/ 10.1175/1520-0442(1998)011<2310:ASFITN > 2.0.CO;2.

Frierson, D. M., 2006: Robust increases in midlatitude static stability in simulations of global warming. Geophys. Res. Lett., 33, L24816, https://doi.org/10.1029/2006GL027504.

Hasselmann, K., 1976: Stochastic climate models Part I. Theory. Tellus, 28, 473-485, https://doi.org/10.3402/tellusa.v28i6.11316.

Hawkins, E., and R. Sutton, 2009: The potential to narrow uncertainty in regional climate predictions. Bull. Amer. Meteor. Soc., 90, 1095-1108, https://doi.org/10.1175/2009BAMS2607.1. 
Jang, C. J., J. Park, T. Park, and S. Yoo, 2011: Response of the ocean mixed layer depth to global warming and its impact on primary production: A case for the North Pacific ocean. ICES J. Mar. Sci., 68, 996-1007, https://doi.org/10.1093/icesjms/ fsr064.

Kosaka, Y., and S. P. Xie, 2013: Recent global-warming hiatus tied to equatorial Pacific surface cooling. Nature, 501, 403-407, https://doi.org/10.1038/nature12534.

Kushnir, Y., 1994: Interdecadal variations in North Atlantic sea surface temperature and associated atmospheric conditions. J. Climate, 7, 141-157, https://doi.org/10.1175/1520-0442(1994) 007<0141:IVINAS >2.0.CO;2.

Li, S., and Coauthors, 2019: The Pacific Decadal Oscillation less predictable under greenhouse warming. Nat. Climate Change, 10, 30-34, https://doi.org/10.1038/s41558-019-0663-x.

Lin, R., F. Zheng, and X. Dong, 2018: ENSO frequency asymmetry and the Pacific decadal oscillation in observations and 19 CMIP5 models. Adv. Atmos. Sci., 35, 495-506, https://doi.org/ 10.1007/s00376-017-7133-z.

Liu, Z., 2012: Dynamics of interdecadal climate variability: A historical perspective. J. Climate, 25, 1963-1995, https://doi.org/ 10.1175/2011JCLI3980.1.

_ decadal surface temperature variation. J. Phys. Oceanogr., 24, 587-608, https://doi.org/10.1175/1520-0485(1994)024<0587: TFBAAD $>2.0 . \mathrm{CO} ; 2$.

MacMartin, D. G., L. Zanna, and E. Tziperman, 2016: Suppression of Atlantic meridional overturning circulation variability at increased $\mathrm{CO}_{2}$. J. Climate, 29, 4155-4164, https://doi.org/ 10.1175/JCLI-D-15-0533.1.

Mantua, N. J., S. R. Hare, Y. Zhang, J. M. Wallace, and R. C. Francis, 1997: A Pacific interdecadal climate oscillation with impacts on salmon production. Bull. Amer. Meteor. Soc., 78, 1069-1080, https://doi.org/10.1175/1520-0477(1997)078<1069: APICOW $>2.0 . \mathrm{CO} ; 2$.
Newman, M., 2007: Interannual to decadal predictability of tropical and North Pacific sea surface temperatures. J. Climate, 20, 2333-2356, https://doi.org/10.1175/JCLI4165.1.

Ortega, P., M. Montoya, F. González-Rouco, J. Mignot, and S. Legutke, 2012: Variability of the Atlantic meridional overturning circulation in the last millennium and two IPCC scenarios. Climate Dyn., 38, 1925-1947, https://doi.org/10.1007/s00382-011-1081-6.

Overland, J. E., and M. Wang, 2007: Future climate of the North Pacific Ocean. Eos, Trans. Amer. Geophys. Union, 88, 178182, https://doi.org/10.1029/2007EO160003.

Saenko, O. A., 2006: Influence of global warming on baroclinic Rossby radius in the ocean: A model intercomparison. J. Climate, 19, 1354 1360, https://doi.org/10.1175/JCLI3683.1.

Schneider, N., and B. D. Cornuelle, 2005: The forcing of the Pacific decadal oscillation. J. Climate, 18, 4355-4373, https://doi.org/ 10.1175/JCLI3527.1.

Wang, J., and C. Li, 2017: Low-frequency variability and possible changes in the North Pacific simulated by CMIP5 models. J. Meteor. Soc. Japan, 95, 199-211, https://doi.org/10.2151/jmsj.2017-011.

Watanabe, M., H. Shiogama, H. Tatebe, M. Hayashi, M. Ishii, and M. Kimoto, 2014: Contribution of natural decadal variability to global warming acceleration and hiatus. Nat. Climate Change, 4, 893-897, https://doi.org/10.1038/nclimate2355.

Wu, S., Z.-Y. Liu, J. Cheng, and C. Li, 2018: Response of North Pacific and North Atlantic decadal variability to weak global warming. Adv. Climate Change Res., 9, 95-101, https://doi.org/ 10.1016/j.accre.2018.03.001.

Yim, B. Y., M. Kwon, H. S. Min, and J.-S. Kug, 2015: Pacific decadal oscillation and its relation to the extratropical atmospheric variation in CMIP5. Climate Dyn., 44, 1521-1540, https://doi.org/10.1007/s00382-014-2349-4.

Zhang, Y., J. M. Wallace, and D. S. Battisti, 1997: ENSO-like interdecadal variability: 1900-93. J. Climate, 10, 1004-1020, https://doi.org/10.1175/1520-0442(1997)010<1004:ELIV>2.0. $\mathrm{CO} ; 2$. 